\title{
Research on Incentive Utility Multi-attribute decision-making Model and its Application
}

\author{
WEN Mi ${ }^{1 *}$, WEN Jing ${ }^{2}$, WU Xiao-feng ${ }^{1}$, ZHANG Yi-ming ${ }^{3}$, FANG Qiang ${ }^{4}$ \\ 1. Unit 95905, Aviation University Air Force, Jinzhou, Liaoning, 121011, China. \\ 2. Staff Room of Training and Management, Institute of NBC Defense of Army, Beijing 102205, China. \\ 3. Unit 92208, Air Force Research Institute, Beijing 100076, China. \\ 4. Unit 952795, Airborne Training Base, Guilin, Guangxi, 541000, China.
}

\begin{abstract}
This paper puts forward the concept of incentive utility, which converts the value of each attribute in multi-attribute decision-making into a unified index for measurement, thus simplifying multi-attribute decision-making. In this paper, a comprehensive evaluation model based on the TOPSIS of incentive utility is established. Through the establishment of incentive index, the utility of incentive and the calculation of ideal point of utility, the disadvantage of "ideal point is not ideal" in the TOPSIS is overcome. It can better synthesize the sub-attribute value and realize the subjective multi-attribute decision-making. The example shows that the model can apply subjective factors to decision-making scientifically, and the result is more reasonable and effective.
\end{abstract}

\section{Introduction}

The multi-value thing widely exists in the real world. In order to determine its value, we must consider how to synthesize the values existing in various attributes, which requires the use of multi-attribute utility theory. In the multi-attribute utility theory, the form of the multiattribute utility function is very complex, and it is generally difficult to obtain, only when certain conditions are satisfied among the attributes (such as the condition of "value independence"). The complex multi-attribute utility function can be expressed in the form of the synthesis of each single-attribute utility function. In reality, it is difficult or even impossible to get the conditions of value independence between attributes, so finding a unified measurement standard and transforming subattribute value into a unified index has become an important research orientation of multi-attribute decisionmaking. According to this idea, this paper puts forward the concept of incentive utility, unifies the index value of each attribute, and the incentive utility index can be applied to multi-attribute decision-making.

\section{The concept of incentive utility}

Berelson and Steiner (American management scientists) define incentive as: "All the conditions, hopes, wishes and motivations that people want to strive for constitute the motivation, it is a kind of inner state of all the activities of people." [1] In this definition, we can see that all human behaviour can be attributed to a certain motivation, which is a mental state that stimulates, promotes and strengthens people's preferred behaviour, and this motivation refers to "incentive". Therefore, incentive is the root cause of people's preference for different things, the size of the preference is determined by the size of the incentive, and the orientation of preference is determined by the orientation of the incentive. For multi-attribute problems, although the values of each attribute are different in dimension and order of magnitude, they can be normalized as incentive.

\subsection{Incentive index}

Incentive is a good index to unify sub-attribute value, but it is very difficult to be quantified reasonably, because of its generality and subjectivity. According to the fairness theory of psychologist Fesdinger [2], the effective motivation to the people comes from the individual's understanding of the fairness in the organization, that is, through the comparison of "horizontal" and "vertical", to judge the degree of fairness of the income. Similarly, when making a trade-off between the two options, decision makers are often faced with the following judgment:

(1) $Q_{A} / I_{A}>Q_{B} / I_{B}$, compared with scheme $\mathrm{B}$, the benefit of scheme $\mathrm{A}$ is larger than that of scheme $\mathrm{B}$, which leads to the positive incentive that decision makers prefer scheme A.

(2) $Q_{A} / I_{A}=Q_{B} / I_{B}$, it means that the two schemes are equivalent and there is no incentive preference, and there is no difference for decision makers to choose scheme $\mathrm{A}$ or scheme B.

In the formula, $I_{A}$ and $Q_{A}$ express the personnel's own feelings about the payments and benefits of scheme

"Corresponding author's e-mail: wenmi2007@sina. 
A, respectively.

"People react to incentives, and the rest is the explanation" [3]. The decision-making preferences can be interpreted as caused by some incentive tendency. The size of the incentive is related to the relative value of the benefits and payments. Incentive value is a good description that affects the preference of decision makers. Incentive has not only size but also direction. If it is stipulated that the incentives that produce favorable effects are positive, the incentives that produce negative effects are negative. Obviously, the direction of motivation is directly related to the positive and negative situation of the difference between their benefits and payments. Therefore, let:

$$
p=Q / I-1=(Q-I) / I
$$

Among them, $I$ is the decision-makers' sense of the reasonable payments of the scheme, and $Q$ is the decision-makers' sense of the benefits of the scheme. $p$ is called incentive index, which can describe the degree of motivation. The higher $p$, the greater the degree of incentive. At that time, when $p>0$, there is a positive incentive, when $p=0$, there is no incentive and when $p<0$, there is a negative incentive. In the actual decision-making, if the reasonable payments are not known, the average value of the payments of each scheme can be used to represent the reasonable payments, that is:

$$
p=(Q-\bar{I}) / \bar{I}
$$

The Formula (1) and (2) are the incentive functions to realize the incentive of the index. Through the incentive of the index, sub-attribute index can be transformed into a unified incentive index.

\subsection{Effectiveness of incentive index}

Just as different decision makers will have different responses to the same consequences, different decision makers may have different preferences for the same incentives, which is related to the cognitive level and risk attitude of decision makers. In order to describe the intensity of the preference of the decision maker, it is necessary to use the utility function corresponding to the decision maker to transform the incentive index into the utility value. Suppose the utility function applicable to the decision maker is $U(x)$, because of the direction of the incentive, the normalized "S" utility function (Formula (3)), can be used to transform the incentive index into the utility value.

$$
U(x)=\frac{1}{1+a e^{-b x}}
$$

In the formula, $a>0, b>0$ are the risk coefficient, and the utility value $U(x)$ represents the degree of preference of the decision maker when the incentive value is $x$. The above process is called the utility of the incentive index, and the incentive index after the utility is called the incentive utility index. Therefore, incentive utility can be defined as:

Define 1: By using the incentive function and the utility function, the decision index can be transform into the incentive utility index. that is:

$$
u_{p}(x)=U(P(x))
$$

Among them, $u_{p}(x), P(\cdot), U(\cdot)$ represent the incentive utility of the index, the incentive function and the utility function. In this paper, formula (1) is used as the incentive function, and formula (3) is the utility function of incentive. The "incentive utility" defined here represents the process of the utility of the incentive index, which is a proper term and is different from the concept of "incentive of utility".

For formula (3), the appropriate setting can be as follows: when the incentive is 1 , the effective value is 0.9 ; when the incentive is 0 , the effective value is 0.5 , that is $U(1)=0.9, U(0)=0.5$. The normalized "S" utility function (In Formula (3), for $a=1, b=2.2$ ) can be described as follows:

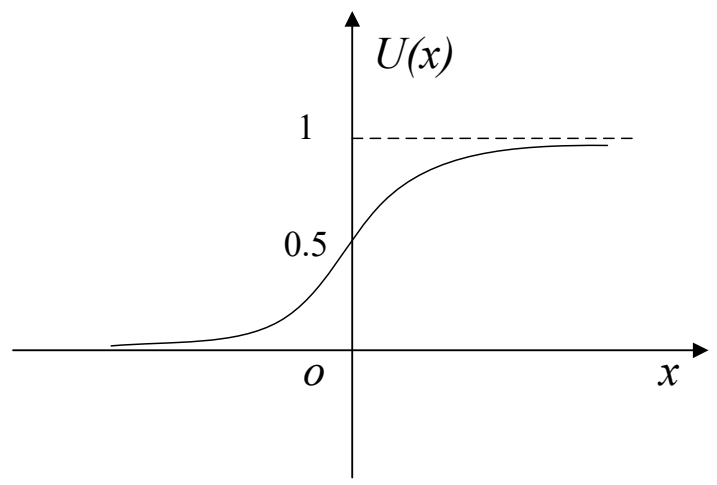

Figure 1. Normalized "S" utility function

As can be seen from figure 1, near the point where the incentive utility value is 0.5 , no matter positive or negative incentive, as long as it is changed a little, the utility value will change greatly. This phenomenon is consistent with the risk aversion psychology of ordinary people in reality. After the incentive and utility of each attribute value, the standardization of multi-attribute index is actually realized. The whole process has two characteristics:

(1) for the incentive function $p=(Q-\bar{I}) / \bar{I}$, it can remove the commonness of the data, while retaining the individual characteristics of the data.

(2) for the "S" utility function(3), the middle is steep, and the point that falling into it is approximately a linear transformation, which will not change the relative distribution position of the point in the data; the two ends are smooth, and the value of the point that falling into it will be compressed rapidly. so that the distribution of points is relatively concentrated.

Therefore, after incentive and utility, the difference of numerical interval is obvious and relatively concentrated, and the final decision result will not be greatly affected by individual data which is too large or too small.

By using the incentive and utility to complete the standardization of the index, the incentive utility index can be directly used in the multi-attribute decision-making model, and form the incentive utility multi-attribute decision-making model. In particular, taking the improved TOPSIS as an example, on the basis of the incentive and 
utility process (realize standardizing of indexes), this paper clarifies the relationship between the ideal point of utility and the ideal point of the scheme, and comprehensively puts forward an evaluation model based on the TOPSIS of incentive utility.

\section{The TOPSIS of incentive utility}

TOPSIS is to find the positive ideal solution and the negative ideal solution of the multi- attribute problem, and take how close the scheme is to the positive ideal solution and how far away from the negative ideal solution, as the basis for evaluating the quality of the scheme [4]. The ideal point (optimal vector and worst vector) selected in the TOPSIS is only the relative ideal point in the scheme domain, not the ideal point in the whole domain, so the traditional ideal solution has the defect that the "ideal point" is not ideal. However, after the incentive and utility of each index in the multi-attribute problem, in addition to making the difference between the obtained numerical intervals more obvious and relatively concentrated, what is more important is that we can get the corresponding utility ideal point $U\left(x^{+}\right)=1$ and $\left.U\left(x^{0}\right)=0\right)$. If the utility ideal point is used instead of the traditional ideal point, the traditional TOPSIS can be improved.

\subsection{Utility ideal point}

For the normalized utility function, $U\left(x^{+}\right)=1$ and $U\left(x^{0}\right)=0$ represent its supremum and infimum respectively (the famous road crossing problem proves the boundedness of utility). In the previous paper, the normalized "S" type utility function is used to unify the utility values to the $(0,1)$ interval, so its corresponding supremum and infimum are 1 and 0 . Obviously, because of the particularity of the normalized "S" utility function, the supremum and infimum can not be obtained. However, $U\left(x^{+}\right)=1$ and $U\left(x^{0}\right)=0$ are meaningful, they represent the most ideal state and the least ideal state in the minds of decision makers (cognitive domain), respectively.

Taking the scheme decision as an example, due to the limitation of cognition and thought, the decision maker will have three different understandings of the ideal scheme: the ideal scheme in the scheme domain, the ideal scheme in the cognitive domain and the actual ideal scheme. Suppose that the positive and negative ideal schemes in the scheme domain are $\mathrm{A}^{+}$and $\mathrm{A}^{-}$, the positive and negative ideal schemes in the cognitive domain are $\mathrm{A}^{+}$ and $\mathrm{A}^{-}$, and the actual positive and negative ideal schemes are $\mathrm{A}^{*}$ and $\mathrm{A}^{\circ}$, as shown in figure 2 .

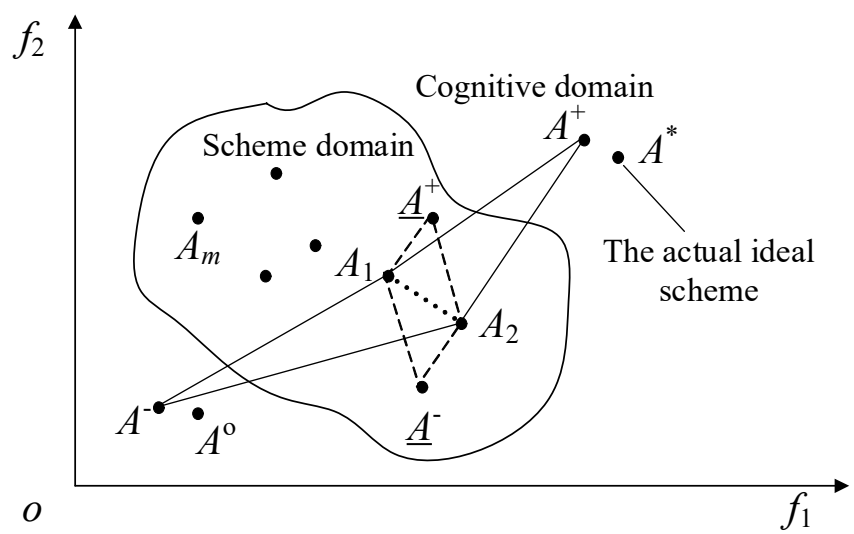

Figure 2. Positive and negative ideal points in each field

Taking the decision-making of the two indicators as an example, we might set the two indicators as the bigger the better. There are $m$ feasible schemes to this problem. The two indicators of each scheme, are recorded as $x_{i 1}$, $x_{i 2}$. Then each scheme $A_{i}$ can be represented by points $A_{i}\left(x_{i 1}, x_{i 2}\right)$ on the plane $f_{1} f_{2}$. Note:

$$
\begin{aligned}
& x_{1}^{*}=\max _{1 \leq i \leq m}\left\{x_{i 1}\right\}, \quad x_{2}^{*}=\max _{1 \leq i \leq m}\left\{x_{i 2}\right\} \\
& x_{1}^{-}=\min _{1 \leq i \leq m}\left\{x_{i 1}\right\}, x_{2}^{-}=\min _{1 \leq i \leq m}\left\{x_{i 2}\right\}
\end{aligned}
$$

Then the positive ideal solution of the scheme domain in this problem is $x_{1}^{*}$ and $x_{2}^{*}$, the negative ideal solution of the scheme domain is $x_{1}^{-}$and $x_{2}^{-}$. Both the positive ideal solution and the negative ideal solution in the scheme domain can be expressed as points $\underline{A}^{+}\left(x_{1}^{*}, x_{2}^{*}\right)$ and $\underline{A}^{-}\left(x_{1}^{-}, x_{2}^{-}\right)$, which are called the positive ideal point and the negative ideal point in the scheme domain, respectively. By the same token, there must be similar ideal points in the cognitive domain (as $A^{+}$and $A^{-}$) and actual domain (as $A^{*}$ and $A^{\circ}$ ) of decision makers. Obviously, there must be the following relationship between the three ideal points:

$$
\begin{gathered}
U\left(\underline{A}^{+}\right) \leq U\left(A^{+}\right)=1, A^{+} \preceq A^{*} \\
U\left(A^{-}\right)=0 \leq U\left(\underline{A}^{-}\right), A^{0} \preceq A^{-}
\end{gathered}
$$

Among them, the total utility function $U(\cdot)$ is generated by the estimation of the scheme domain, and the difference between the ideal points in the cognitive domain and the actual domain is determined by the degree of cognition. The higher the degree of cognition, the smaller the difference between them. The decisionmaking according to the utility ideal point (cognitive domain ideal point) is more credible. Therefore, the practical significance of $U\left(x^{+}\right)=1, U\left(x^{o}\right)=0$ is that the ideal point in the scheme domain can be extended to the cognitive domain, and the ideal point of the scheme can be 
replaced by the utility ideal point, which can greatly improve the multi-attribute decision-making model based on the ideal point technology.

\subsection{Modeling}

If there are $m$ schemes as $A_{1} \sim A_{m}$, each scheme has $n$ decision attributes as $X_{1} \sim X_{m}$. They constitute a decision information table, as shown in Table 1.

Table1. Decision information table

\begin{tabular}{ccccc}
\hline \multirow{2}{*}{ Targets/Schemes } & \multicolumn{3}{c}{ Index } \\
\cline { 2 - 5 } & $f_{1}$ & $f_{2}$ & $\cdots$ & $f_{n}$ \\
\hline$A_{1}$ & $x_{11}$ & $x_{12}$ & $\cdots$ & $x_{1 n}$ \\
$A_{2}$ & $x_{21}$ & $x_{22}$ & $\ldots$ & $x_{2 n}$ \\
$\vdots$ & $\vdots$ & $\vdots$ & $\vdots$ & $\vdots$ \\
$A_{m}$ & $x_{m 1}$ & $x_{m 2}$ & $\cdots$ & $x_{m n}$ \\
\hline
\end{tabular}

The matrix $X=\left(x_{i j}\right)_{m \times n}$ composed of each attribute index is called the decision matrix, $A_{i}=\left(x_{i 1}, x_{i 2}, \cdots, x_{i n}\right)$ is the $i$-th scheme, $\underline{A}^{+}=\left(x_{1}^{+}, x_{2}^{+}, \cdots, x_{3}^{+}\right)$and $\underline{A}^{-}=\left(x_{1}^{-}, x_{2}^{-}, \cdots, x_{n}^{-}\right)$represents the positive and negative ideal schemes in the scheme domain, respectively. The definition of relative closeness is as follows:

Define 2: The relative closeness of the scheme $A_{i}=\left(x_{i 1}, x_{i 2}, \cdots, x_{i n}\right)$ can be expressed as:

$$
u_{1}^{+}=D_{i}^{-} /\left(D_{i}^{+}+D_{i}^{-}\right)
$$

$$
\text { Among them: } \quad D_{i}^{+}=\sqrt{\sum_{j=1}^{n}\left(x_{i j}-x_{j}^{+}\right)^{2}}
$$$$
D_{i}^{-}=\sqrt{\sum_{j=1}^{n}\left(x_{i j}-x_{j}^{-}\right)^{2}}(i=1,2, \cdots, m) \text {. }
$$

The relative closeness degree represents the synthesis of the closeness degree between the scheme and the positive ideal scheme and the far away degree of the negative ideal scheme, and describes the "ideal" degree of the scheme, so the greater the closeness degree, the better the scheme. Under the relative closeness criterion, by using the incentive utility index and replacing the ideal point in the scheme domain with the utility ideal point, the TOPSIS of incentive utility can be obtained.

Define 3: For a multi-attribute decision-making problem with $m$ schemes and $n$ indexes, the initial decision matrix is $X=\left(x_{i j}\right)_{m \times n}$, and the incentive utility matrix is $Y=\left(y_{i j}\right)_{m \times n}$, which is obtained through incentive and utility. if the weight of each index is $W=\left(\omega_{1}, \omega_{2}, \cdots, \omega_{n}\right)^{\prime}$, the weighted incentive utility matrix $Z=\left(z_{i j}\right)_{m \times n}=\left(\omega_{j} y_{i j}\right)_{m \times n}$ can be obtained, as the final decision matrix. Replace the ideal point of the scheme with the utility ideal point, that is $Z^{+}=\left(\omega_{1} y_{1}^{+}, \omega_{2} y_{2}^{+}, \cdots \omega_{n} y_{n}^{+}\right)=\left(\omega_{1}, \omega_{2}, \cdots, \omega_{n}\right) \quad$ and
$Z^{-}=\left(\omega_{1} y_{1}^{-}, \omega_{2} y_{2}^{-}, \cdots \omega_{n} y_{n}^{-}\right)=(0,0, \cdots, 0)$, if $A_{1}, A_{2}$ are two schemes in the scheme domain, $u_{1}^{+}, u_{2}^{+}$represent the relative closeness (scheme $A_{1}, A_{2}$, respectively) to the utility ideal point of the scheme (under the weighted incentive utility matrix Z). If $u_{1}^{+}>u_{2}^{+}$, it is said that the scheme $A_{1}$ is better than $A_{2}$ under the relative closeness criterion, marked as: $A_{1} \succ A_{2}$.it can be described as follows:

$$
u_{1}^{+}>u_{2}^{+} \Leftrightarrow A_{1} \succ A_{2}
$$

If the relative closeness of the two schemes $\left(A_{1}\right.$ and $\left.A_{2}\right)$ are the same $\left(u_{1}^{+}=u_{2}^{+}\right)$, it is considered that there is no difference between the two schemes, that is $A_{1} \sim A_{2}$, there are:

$$
u_{1}^{+}=u_{2}^{+} \Leftrightarrow A_{1} \sim A_{2}
$$

If there is a scheme $A_{s} \in A$, which is $u_{s}^{+}=\max _{i=1}^{m}\left\{u_{i}^{+}\right\}$, then the scheme is called the optimal scheme under the relative closeness criterion, which is the evaluation model based on the ideal solution of incentive utility.

\subsection{Solution of the model}

The solving process of the TOPSIS of incentive utility is roughly divided into three steps [5]:

(1) the incentive utility matrix is generated by the incentive and utility;

(2) obtain the weight of the index, and then form a weighted incentive utility matrix;

(3) replace the positive and negative ideal points in the scheme domain with utility ideal points, Calculate the relative closeness, and sort according to the relative closeness, get the optimal scheme.

The whole solution process is called "the TOPSIS of incentive utility", and the flow of the algorithm can be abstracted as figure 3 . 


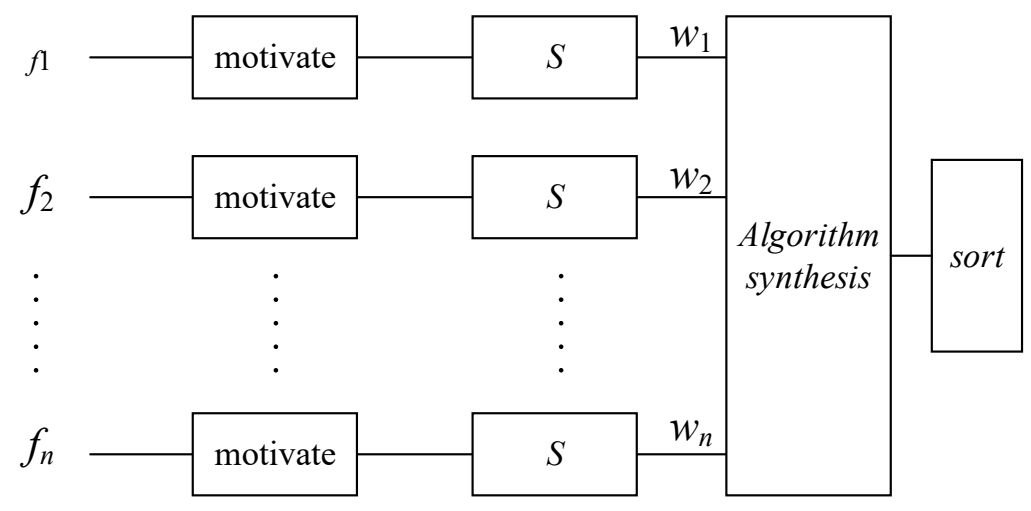

Figure 3. Flow chart of ideal solution of incentive utility

(1) Incentive utility matrix

According to the direction of decision-making indexes, decision-making index can be divided into three categories: benefit (positive) index, cost (reverse) and intermediate index. In the decision matrix $X=\left(x_{i j}\right)_{m \times n}$, the use of formula (2) to transform them into the corresponding incentive indicators:

(1) for the benefit-oriented index $f_{j}$, take $\bar{x}_{j}=\frac{1}{m} \sum_{i=1}^{m} x_{i j} \quad, \quad$ then: $\quad p_{i j}=\frac{x_{i j}-\bar{x}_{j}}{\bar{x}_{j}}$, $(1 \leq i \leq m, 1 \leq j \leq n)$;

(2) for the cost-based index $f_{j}$, take $\bar{x}_{j}=\frac{1}{m} \sum_{i=1}^{m} x_{i j}$, then: $p_{i j}=-\frac{x_{i j}-\bar{x}_{j}}{\bar{x}_{j}}, \quad(1 \leq i \leq m, 1 \leq j \leq n)$;

(3) for the intermediate index $f_{j}$, take $\bar{x}=$ Ideal Value, then: $p_{i j}=-\left|\frac{x_{i j}-\bar{x}}{\bar{x}}\right|, \quad(1 \leq i \leq m, 1 \leq j \leq n)$.

After the above transformation, no matter the positive (benefit) index, the reverse (cost) index or the intermediate index are transformed into positive decision-making index. After transformation, $p_{i j} \in(-\infty,+\infty)$, the matrix $P=\left(p_{i j}\right)_{m \times n}$ is called incentive matrix. Use formula (3)

$$
\begin{gathered}
Z^{+}=\left(z_{1}^{+}, z_{2}^{+}, \cdots, z_{n}^{+}\right)=\left(\omega_{1} y_{1}^{+}, \omega_{2} y_{2}^{+}, \cdots \omega_{n} y_{n}^{+}\right)=\left(\omega_{1}, \omega_{2}, \cdots, \omega_{n}\right) \\
Z^{-}=\left(z_{1}^{-}, z_{2}^{-}, \cdots, z_{n}^{-}\right)=\left(\omega_{1} y_{1}^{-}, \omega_{2} y_{2}^{-}, \cdots \omega_{n} y_{n}^{-}\right)=(0,0, \cdots, 0)
\end{gathered}
$$

Suggest the distances from the $i$-th scheme to the positive utility ideal scheme $Z^{+}$and to the negative utility ideal scheme $Z^{-}$are $D_{i}^{+}, D_{i}^{-}$:

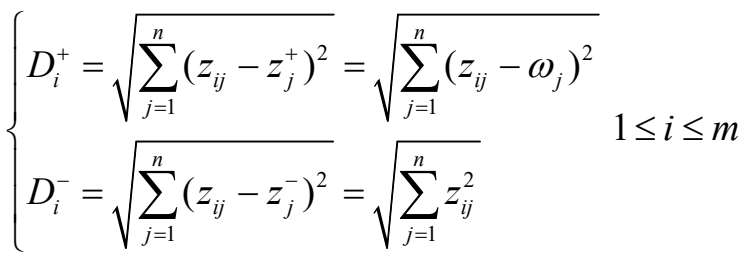

According to formula (5), the relative closeness of each scheme is calculated in turn. According to the principle of "the most is the best", the relative closeness is sorted, and the decision result is obtained. to make the incentive matrix utility.

$$
y_{i j}=U\left(p_{i j}\right)=\frac{1}{1+a e^{-b p_{i j}}}
$$

The incentive utility matrix $Y=\left(y_{i j}\right)_{m \times n}$ is obtained, which satisfies $0<y_{i j}<1$ and is a positive index, the supremum is 1 and the infimum is 0 .

(2) Weighted incentive utility matrix

The weights of each index are as follows:

$$
W=\left(\omega_{1}, \omega_{2}, \cdots, \omega_{n}\right)^{\prime}
$$

Among them, $\omega_{j}$ represents the weight of the $j$-th index. As a result, the weighted incentive utility matrix can be obtained:

$$
Z=\left(z_{i j}\right)_{m \times n}=\left(\omega_{j} y_{i j}\right)_{m \times n}
$$

(3) Calculate the relative closeness and sort it.

Obviously, the positive and negative ideal points of $Y$ are:

$$
\begin{gathered}
Y^{+}=\left(y_{1}^{+}, y_{2}^{+}, \cdots, y_{n}^{+}\right)=(1,1, \cdots, 1) \text { and } \\
Y^{-}=\left(y_{1}^{-}, y_{2}^{-}, \cdots, y_{n}^{-}\right)=(0,0, \cdots, 0)
\end{gathered}
$$

Among them, $y_{j}^{+}$is the positive utility ideal value of the $j$-th index and $y_{j}^{-}$is the negative utility ideal value of the $j$-th index. Therefore, the ideal points of positive and negative utility of weighted incentive utility matrix are:

\section{The application of the TOPSIS of incentive utility}

In order to configure a production system, it is necessary to comprehensively consider the factors such as system efficiency, cost, risk, adaptability, compatibility and effectiveness (marked as $f_{1} \sim f_{6}$ ). There are seven configuration schemes (expressed in terms of $X_{1} \sim X_{7}$ ), and the index values of each scheme are shown in Table 2. It is necessary to make a comprehensive comparison of various factors to select the optimal configuration scheme. 
Table2. Index values of each configuration fee scheme

\begin{tabular}{ccccccc}
\hline Index/ Scheme & Efficiency & Cost & Risk & Adaptability & Compatibility & Validity \\
\hline X1 & 1.0720 & 3060 & 0.64 & 46.373 & 2.0940 & 0.974 \\
X2 & 1.4420 & 3050 & 0.64 & 38.721 & 2.0850 & 0.974 \\
X3 & 0.7180 & 3032 & 0.72 & 34.943 & 1.4080 & 0.974 \\
X4 & 0.7050 & 3025 & 0.74 & 31.256 & 1.4080 & 0.981 \\
X5 & 0.9860 & 3900 & 0.25 & 33.273 & 1.4080 & 0.994 \\
X6 & 0.4939 & 1732 & 0.17 & 43.215 & 1.9858 & 0.946 \\
X7 & 0.9909 & 1145 & 0.22 & 30.732 & 1.9858 & 0.946 \\
\hline
\end{tabular}

Among them, the decision-making index $f_{1}, f_{4}, f_{5}, f_{6}$ are benefit-oriented index, $f_{2}, f_{3}$ are costoriented index. Using AHP method to get the weight of each index is $W=\left(\begin{array}{llllll}0.3 & 0.17 & 0.13 & 0.15 & 0.11 & 0.14\end{array}\right)^{T}$. In formula (2), for $a=1, b=2.2$, the incentive utility matrix is obtained, as shown in Table 3 .

Table3. Incentive utility matrix of the index values of each configuration fee scheme

\begin{tabular}{ccccccc}
\hline Index/ Scheme & Efficiency & Cost & Risk & Adaptability & Compatibility & Validity \\
\hline X1 & 0.593 & 0.4286 & 0.3283 & 0.637 & 0.6001 & 0.5023 \\
X2 & 0.78 & 0.4306 & 0.3283 & 0.5266 & 0.5974 & 0.5023 \\
X3 & 0.3836 & 0.4342 & 0.2534 & 0.4704 & 0.3899 & 0.5023 \\
X4 & 0.3762 & 0.4356 & 0.2366 & 0.4163 & 0.3899 & 0.5063 \\
X5 & 0.5423 & 0.2748 & 0.7429 & 0.4457 & 0.3899 & 0.5137 \\
X6 & 0.2664 & 0.6883 & 0.8062 & 0.5925 & 0.5674 & 0.4865 \\
X7 & 0.5452 & 0.7806 & 0.7681 & 0.4087 & 0.5674 & 0.4865 \\
\hline
\end{tabular}

Calculate the relative closeness of each scheme:

$$
U^{+}=(0.5394,0.6043,0.4042,0.3936,0.4956,0.4646,0.5756)
$$

The ranking result of each scheme is: $X_{2}>X_{7}>X_{1}>X_{5}>X_{6}>X_{3}>X_{4}$. The decision maker should adopt the scheme $X_{2}$. However, according to the sum of the rows of the weighted utility matrix $Z=\left(z_{i j}\right)_{m \times n}$, the decision result is $X_{7}$. It can be seen that the TOPSIS of incentive utility can overcome the interference among multi-quality factors, which is better than the decision-making criterion of directly weighted total utility.

\section{Conclusion}

In this paper, the concept of incentive utility is proposed and applied to multi-attribute decision-making. In particular, combined with the boundedness of utility, the ideal point in the scheme domain is extended to the ideal point in the cognitive domain by using the utility ideal point, and the TOPSIS of incentive utility model is established. It overcomes the shortcomings of TOPSIS, such as the ideal point is not ideal, the standardization is out of proportion and deliberately avoids subjective factors, and the specific algorithm flow is given. It is found that the combination of utility theory and common multiattribute decision-making method is feasible, and the combined method can be better applied to practical decision-making.

\section{References}

1. Gao, J.P. (2008) Research on College Young Teachers' Demand Characteristics and Drive Mechanism, Consume Guide, 4:158.

2. Li A.M. (2007) A Utility-based Model of Civil Servant's Motivation and Its application. Huazhong University of Science and Technology, Wuhan.

3. Mankiw, N.G. (2006) Principles of Economics (Fourth Edition): Fascicles of Microeconomics. Peking University Press, Beijing.

4. Ge, Y., Liu, S.T. (2020) Jamming Effectiveness Evaluation of Radar Seeker Based on Vague Set and TOPSIS Method, Journal of CAEIT, 15: 364-369.

5. Wang, J.S, Wen, M. (2011) Ideal Solving Method Based on Motivation Utility Function, Journal of Chongqing University of Technology (Nature Science), 25:116-119.

6. Yang, W.G, Shang, H., Luo, Z. (2020) Research on National Urban Emergence Management Capability Assessment under Improved TOPSIS Method, Mathematics in Practice and Theory, 50:21-28.

7. Chen, X., Wei, H. (2020) Research on product design evaluation based on TOPSIS method of structure entropy weight, Journal of Graphics, 41: 446-452.

8. Wang, M.X., Zhai, H.L. (2020) Evaluation method and application rehabilitation training products for 
autistic children based on AHP and TOPSIS, Journal of Graphics, 41: 453-460.

9. Hao, X.M., Li, L.L., Li, M.W., et al. (2020) TOPSIS Method Based on Mixed Fuzzy Numbers and Evaluation of Overcapacity, Mathematics in Practice and Theory, 50: 47-56.

10. Zhou, Y. (2009) The Study of TOPSIS Method in Multiple Attribute Decision Making. Wuhan University of Technology, Wuhan.

11. Zhang, H.M., Li, X.N. (2020) Hesitant Fuzzy Multiattribute Decision Making Based on Conflict Analysis, Pattern Recognition and Artificial Intelligence, 33: 610-618.

12. Xu, X.H., Zhao, C.W., He, J.S., et al. (2020) Association variable weight space multi-attribute decision method under multi-type heterogeneous data, Systems Engineering-Theory \& Practice, 40: 18961904. 